\title{
Sperm binding capacity and ultrastructure of the zona pellucida of stored canine oocytes
}

\author{
B. Ström Holst, B. Larsson, C. Linde-Forsberg* and H. Rodriguez-Martinez \\ Department of Obstetrics and Gynaecology, Box 7039, Faculty of Veterinary Medicine, Swedish University of Agricultural Sciences \\ (SLU), Centre for Reproductive Biology in Uppsala, SE-750 07 Uppsala, Sweden
}

\begin{abstract}
Sperm binding to the zona pellucida is a prerequisite for fertilization, and tests that evaluate this function have been described for several species. When carrying out such tests in the canine species, ovaries or oocytes have to be stored to obtain a sufficient number of oocytes at the time of testing. In the present study, the sperm binding capacities of salt-stored oocytes and oocytes from deep frozen ovaries were measured and compared with that of fresh oocytes. Two different procedures for washing the sperm-oocyte complexes (gentle and tough) were used before evaluating the number of bound spermatozoa. The total number of oocytes that bound spermatozoa was significantly lower for both salt-stored and deep frozen oocytes compared with fresh oocytes. Significantly fewer spermatozoa bound to stored oocytes than to fresh oocytes $(P \leqslant 0.05)$ irrespective of washing procedure and there was no significant difference between the two methods of storage. Scanning electron microscopy revealed that the zona pellucida of fresh oocytes was sponge-like in appearance with fenestrations surrounded by a meshwork of filaments. In stored oocytes, the meshwork tended to be wider, with larger fenestrations. The thickness of the zona pellucida was $0.3-1.0 \mu \mathrm{m}$ in salt-stored oocytes and 6-8 $\mu \mathrm{m}$ in oocytes from frozen ovaries, whereas it was 3-6 $4 \mathrm{~m}$ in fresh oocytes. Changes in morphology that occur under the various storage conditions tested might have contributed to the reduced sperm binding capacity of the stored oocytes. Although stored canine oocytes do not bind as many spermatozoa as do fresh oocytes, the former can be used in a zona pellucida binding assay, and the deep freezing of the ovaries appears to be a better storage method than storing oocytes in salt solution.
\end{abstract}

\section{Introduction}

The binding of spermatozoa to the zona pellucida is an initial and crucial step in fertilization. Studies of sperm-zona pellucida interactions have been used both for understanding details of sperm action at fertilization and for assessing sperm fertilizing potential. Tests to determine the capacity of spermatozoa to bind to and penetrate intact zonae pellucidae or hemizonae have been applied in the canine species (Hay et al., 1997a,b; Mayenco-Aguirre and Pérez Cortés, 1998). The results suggest that these tests are useful for evaluating canine sperm function.

A constant source of oocytes must be available if tests based on sperm-oocyte interactions are to be practical. Canine oocytes can be obtained from ovaries removed in connection with an ovariohysterectomy (B. Ström Holst, B. Larsson, H. Rodriguez-Martinez, A-S. Lagerstedt and C. Linde-Forsberg, unpublished). If the ovariohysterectomies are performed infrequently, ovaries or oocytes need to be

${ }^{*}$ Correspondence.

Received 7 July 1999. stored. Before oocyte recovery for a zona pellucida binding test, ovaries can be kept chilled, for short-term storage, or frozen for long-term storage. Another possible method of long-term storage is to keep recovered oocytes in a salt solution. In other studies evaluating canine sperm binding to the zona pellucida, fresh or salt-stored cumulus-oocyte complexes (COCs) from fresh ovaries (Hay et al., 1997a,b), COCs from chilled ovaries (Hay et al., 1997b), frozen oocytes from fresh ovaries (Ivanova et al., 1999) and frozen oocytes from frozen ovaries (Mayenco-Aguirre and Pérez Cortés, 1998) have been used. Sperm attachment and penetration capacities did not differ significantly between COCs from fresh and chilled ovaries (Hay et al., 1997a). However, the number of spermatozoa able to attach to or penetrate the zona pellucida was significantly lower for salt-stored COCs compared with fresh COCs (Hay et al., 1997a). The zonae pellucidae of frozen oocytes from frozen ovaries have been used to discriminate fertile from infertile dogs in a hemizona binding assay (Mayenco-Aguirre and Pérez Cortés, 1998).

Other studies have reported that the ultrastructure of the zona pellucida changes after fertilization in humans and cows (Nikas et al., 1994, Suzuki et al., 1994). These ultrastructural 
changes were thought to correspond to the zona reaction. Henkel et al. (1995) also reported that the functional ability of the zona pellucida to bind spermatozoa is closely connected to its morphological appearance. Since oocyte storage may cause ultrastructural changes in the zona pellucida, ultrastructural examination of stored oocytes might be helpful in assessing the usefulness of different storage methods. An ultrastructural change in the zona pellucida might also affect the relation between loosely and tightly bound spermatozoa.

The aim of the present study was to evaluate the effect of different methods of storing canine oocytes on the outcome of a sperm-zona pellucida binding assay and on the ultrastructure of the zona pellucida.

\section{Materials and Methods}

\section{Zona pellucida binding assay}

Oocyte preparation. Oocytes from six bitches $(7.5 \pm 1.0$ years old) subjected to ovariohysterectomy at the Department of Small Animal Clinical Sciences, SLU, Sweden, were used for this study. The ovaries were collected within $1 \mathrm{~h}$ after ovariohysterectomy and were washed with physiological saline. From one of the ovaries (FRESH) the oocytes, which had been stored for no longer than $5 \mathrm{~h}$ in physiological saline at $4^{\circ} \mathrm{C}$, were recovered by mincing the ovary with a scalpel in a Petri dish with PBS with $0.5 \%(\mathrm{w} / \mathrm{v})$ BSA under a stereo microscope. The cumulus cells surrounding the oocytes were removed by incubating the COCs in $75 \mathrm{mmol}$ sodium citrate $\mathrm{1}^{-1}$ for $15 \mathrm{~min}$, followed by vortexing (Vortex Genie 2, Labora, Sollentuna) for $15 \mathrm{~min}$. The other ovary (FROZEN) was stored in $20 \mathrm{ml} \mathrm{NaCl}$ without additives for 1 week at $-20^{\circ} \mathrm{C}$ and after thawing at room temperature the oocytes were recovered and the cumulus cells removed as described above. Oocytes were assigned to one of the following treatments:

Fresh oocytes (FRE). Half of the oocytes recovered from the fresh ovaries were stored overnight at $4^{\circ} \mathrm{C}$ in PBS with $0.5 \%$ $(\mathrm{w} / \mathrm{v}) \mathrm{BSA}$, and used the next day for a sperm-zona pellucida binding assay.

Salt-stored oocytes from fresh ovaries (FRESS). The remaining oocytes recovered from the fresh ovaries were stored for 1 week at $4^{\circ} \mathrm{C}$ in a salt solution consisting of $0.5 \mathrm{~mol}\left(\mathrm{NH}_{4}\right)_{2} \mathrm{SO}_{4} \mathrm{l}^{-1}, 0.75 \mathrm{~mol} \mathrm{MgCl}_{2} \mathrm{l}^{-1}, 0.2 \mathrm{mmol} \mathrm{ZnCl}_{2} \mathrm{l}^{-1}$ and $0.1 \mathrm{mg}$ polyvinyl alcohol $\mathrm{ml}^{-1}$ (Boatman et al., 1988) with 40 mmol HEPES buffer $1^{-1}$, pH 7.4 (Andrews et al., 1992). The oocytes were washed $(6 \times 30 \mathrm{~min})$ in $3 \mathrm{ml}$ PBS with $0.5 \%$ $(\mathrm{w} / \mathrm{v})$ BSA and used in a sperm-zona pellucida binding assay.

Oocytes from frozen ovaries (FRO). After the cumulus cells had been removed, the oocytes were placed in PBS with $0.5 \%$ $(\mathrm{w} / \mathrm{v})$ BSA and used the same day for a sperm-zona pellucida binding assay.

\section{Semen preparation}

The first and second fractions of one ejaculate from one dog were collected and subjectively evaluated for progressive motility at $\times 400$ magnification in a phase-contrast microscope. After centrifugation at $700 \mathrm{~g}$ for $8 \mathrm{~min}$, the sperm pellet was extended in two steps and frozen using a Tris-citrate-glucose extender with $0.5 \%$ (v/v) Equex STM paste added at a concentration of $50 \times 10^{6}$ spermatozoa per $0.5 \mathrm{ml}$ straw (Rota et al., 1997).

Semen was thawed in a $70^{\circ} \mathrm{C}$ waterbath for $8 \mathrm{~s}$ and then held at $37^{\circ} \mathrm{C}$ for $5 \mathrm{~min}$. Motility after thawing was assessed, and straws with $\geqslant 70 \%$ motile spermatozoa were used in the sperm-zona pellucida binding assay.

Thawed semen $(400 \mu \mathrm{l})$ from each straw was gently poured on top of a Percoll column ( $1 \mathrm{ml}$ of $80 \%(\mathrm{v} / \mathrm{v})$ Percoll and $1 \mathrm{ml}$ of $40 \%(\mathrm{v} / \mathrm{v})$ Percoll in canine capacitation medium (CCM; Mahi and Yanagimachi, 1978) without antibiotics) and centrifuged for $30 \mathrm{~min}$ at $400 \mathrm{~g}$. The supernatant was discarded, the volume of the pellet was measured and the pellet was diluted 1:10 in CCM. After $5 \mathrm{~min}$ at $37^{\circ} \mathrm{C}$ the motility and concentration of the sample were assessed, and CCM was then added to reach a final concentration of $0.625 \times 10^{6}$ spermatozoa $\mathrm{ml}^{-1}$. Samples with a motility of $\geqslant 75 \%$ were included.

\section{Sperm-zona pellucida binding}

Droplets $(10 \mu \mathrm{l})$ of CCM were prepared in a small Petri dish $(35 \mathrm{~mm} \times 10 \mathrm{~mm})$ and covered with $3 \mathrm{ml}$ mineral oil. One to five oocytes were added per droplet and incubated in $5 \% \mathrm{CO}_{2}$ in air at $37^{\circ} \mathrm{C}$ and $100 \%$ humidity for a minimum of $1 \mathrm{~h}$. Sperm suspension $(40 \mu \mathrm{l})$ was added to the droplet, resulting in a final concentration of $0.5 \times 10^{6}$ spermatozoa $\mathrm{ml}^{-1}$ in a $50 \mu \mathrm{l}$ droplet. The droplets with spermatozoa and oocytes were incubated for $4 \mathrm{~h}$. After incubation, the oocytes were transferred to $100 \mu \mathrm{l}$ droplets of PBS with $0.5 \%(\mathrm{w} / \mathrm{v})$ BSA and pipetted ten times using a $0.7 \mathrm{~mm}$ diameter pipette (gentle wash) to remove loosely attached spermatozoa. The washed oocytes were fixed for $15 \mathrm{~min}$ in $1.5 \%(\mathrm{v} / \mathrm{v})$ glutaraldehyde in a $0.067 \mathrm{~mol}$ sodium cacodylate $\mathrm{l}^{-1}$ buffer and stored in PBS with $0.5 \%(\mathrm{w} / \mathrm{v})$ BSA until evaluation. A total of 178 oocytes, FRE, FRESS and FRO, were used in the sperm-zona pellucida binding assays.

Spermatozoa bound to the zona pellucida were counted after staining the sperm-oocyte complexes for 15 min with a solution of $10 \mu \mathrm{l}$ propidium iodide in $500 \mu \mathrm{l}$ PBS with BSA. The counts were made using epifluorescence UV illumination on a Diaplan Leitz microscope $(\times 160-400)$ by placing the stained sperm-oocyte complex, without a cover glass, on an excavated glass slide. After counting the spermatozoa, the oocytes were subjected to a second wash (tough wash), performed by pipetting ten times in a $100 \mu \mathrm{l}$ droplet of PBS using a pipette the inner diameter of which had been manually adjusted to be only slightly larger than the oocyte (0.1-0.2 mm). After pipetting, bound spermatozoa were counted again.

\section{Ultrastructural study}

Ovaries from an additional seven bitches $(5.9 \pm 2.8$ years old) subjected to ovariohysterectomy were used for an ultrastructural study of the zona pellucida. The ovaries were 
collected within $1 \mathrm{~h}$ after ovariohysterectomy and were washed with physiological saline. The ovaries and oocytes from each bitch were assigned to one of four treatments, as described below.

From one ovary (FRESH) oocytes were recovered immediately, as described above, and the cumulus cells were removed. The other ovary (FROZEN) was stored in $20 \mathrm{ml} \mathrm{NaCl}$ without any cryoprotectant or other additive at $-20^{\circ} \mathrm{C}$ for 1 week and thawed at room temperature. The denuded oocytes from the FRESH ovary were divided into two groups, that is FRE and FRESS. The oocytes in the FRE group were washed three times in PBS and fixed in 2\% (v/v) glutaraldehyde in 0.1 mol sodium cacodylate buffer $1^{-1}$. The other oocytes (FRESS) were stored for 1 week at $4^{\circ} \mathrm{C}$ in the salt storage solution used for the sperm-zona pellucida binding assay. After 1 week, the FRESS oocytes were washed in PBS $(6 \times 30-60 \mathrm{~min})$ and fixed in the same way as the FRE group. Oocytes from the FROZEN ovary were recovered, the cumulus cells were removed as described earlier, and the denuded oocytes were divided into two groups, that is FRO and FROSS. Oocytes in the FRO group were fixed immediately, whereas those in the FROSS group were stored in a salt solution for 1 week at $4^{\circ} \mathrm{C}$, washed $(6 \times 30-60 \mathrm{~min})$ in PBS and fixed. FROSS oocytes were not included in the sperm-zona pellucida binding assay, but were added to the ultrastructural study to make the experimental design complete.

All oocytes were subjected to a second fixation in $2 \%(\mathrm{w} / \mathrm{v})$ osmium tetroxide and dehydrated in increasing concentrations of acetone. After being subjected to critical-point drying, the oocytes were mounted on stubs and sputtered with platinumpalladium. The analysis was performed using a SEM JEOL 6320F scanning electron microscope (JEOL Ltd, Tokyo).

A total of 104 oocytes (FRE, FRESS, FRO and FROSS) were examined. Oocyte diameter, thickness of the zona pellucida (measured in damaged oocytes) and morphology of the zona pellucida were evaluated from micrographs.

\section{Statistical analysis}

Zona pellucida binding assay. The statistical analysis was carried out with a generalized linear mixed model in SAS GLIMMIX macro (Littell et al., 1996) in the SAS statistical package (SAS version 6.12, SAS Institute Inc., Cary, NC). The model used was:

$$
y_{\mathrm{ijkl}}=\mu+\alpha_{\mathrm{i}}+\beta_{\mathrm{j}}+d_{\mathrm{k}}+a b d_{\mathrm{ijk}}+e_{\mathrm{ijkl}}
$$

where $\alpha_{i}$ and $\beta_{j}$ were the fixed effects of the type of oocyte treatment (fresh oocytes versus oocytes from frozen ovaries versus salt-stored oocytes) and the method of washing of the sperm-egg complexes (gentle or tough); $d_{\mathrm{k}}$ and $a b d_{\mathrm{ijk}}$ were random effects of animals and their interaction with the fixed effects, assumed to be independent and normally distributed. $e_{\mathrm{ijkl}}$ was assumed to follow a Poisson distribution and the data were transformed to $\log _{10}$ values for the calculations. A model including the interactions between the fixed treatment effects was evaluated first, but since these interactions were not significant, they were excluded (Altman, 1991). $P \leqslant 0.05$ was set as the level of statistical significance. The results are presented as back-transformed means.
Chi-squared analysis was used to compare the different treatments in terms of the proportion of oocytes with bound spermatozoa using the Minitab statistical software (Minitab Inc., State College, PA); the level of statistical significance was set to $P<0.05$.

Ultrastructural study. The diameters of the oocytes in the different treatment groups were compared using the Mann-Whitney U test (Minitab Inc., State College, PA) and the significance level was set at $P<0.05$. Values are presented as means $\pm \mathrm{SD}$.

\section{Results}

\section{Sperm-zona pellucida binding assay}

There was a significant effect of oocyte treatment on the number of spermatozoa bound to the zona pellucida $(P<0.001)$. The number of spermatozoa that bound to unstored oocytes from fresh ovaries (FRE) was significantly higher compared with the number binding to salt-stored oocytes (FRESS) or to oocytes from frozen stored ovaries (FRO) $(P<0.001)$. There was no significant difference between the number of spermatozoa that bound to FRESS oocytes and the number bound to FRO oocytes. Significantly fewer spermatozoa remained bound after the tough wash than after the gentle wash $(P<0.001)$ for all treatment groups except the salt-stored oocytes (FRESS) $(P<0.2)$. However, there was no significant interaction between oocyte treatment and type of wash.

There was a significant effect $(P<0.01$ for the gentle wash and $P<0.05$ for the tough wash) of oocyte treatment on the proportion of oocytes that had no spermatozoa bound to them. For the gentle wash, the number of oocytes with spermatozoa attached to the zona pellucida was significantly lower for the FRESS group than for FRE group $(P<0.02)$, whereas the difference between FRE and FRO oocytes was not significant. For the tough wash, the proportion of oocytes with spermatozoa bound to the zona pellucida was significantly lower for both FRESS and FRO groups compared with the FRE group $(P<0.02)$. The results are summarized (Table 1).

\section{Ultrastructural study}

No relationship could be found between the age of the bitch and morphology of the zona pellucida, nor was there any obvious relationship between ovarian morphology (presence of follicles or corpora lutea) and morphology of the zona pellucida. There was no significant difference in the diameter of oocytes from different storage treatments. There was a large variation in the morphology of the surface of the zona pellucida both within individual bitches and within treatment groups.

Oocytes from fresh ovaries (FRE). Most oocytes had a zona pellucida characterized by a fibrous network with numerous fenestrations of intermediate size (Fig. 1a,b). Oocytes with a 
Table. 1. Number of canine spermatozoa bound to fresh and stored canine oocytes after gentle and tough washing of the sperm-oocyte complexes

\begin{tabular}{|c|c|c|c|c|c|}
\hline \multirow[b]{2}{*}{$\begin{array}{l}\text { Oocyte } \\
\text { treatment }\end{array}$} & \multirow[b]{2}{*}{$\begin{array}{c}\text { Number of } \\
\text { oocytes }\end{array}$} & \multicolumn{2}{|c|}{ Gentle washing procedure } & \multicolumn{2}{|c|}{ Tough washing procedure } \\
\hline & & $\begin{array}{c}\text { Number of } \\
\text { spermatozoa per } \\
\text { oocyte }\end{array}$ & $\begin{array}{c}\text { Percentage of } \\
\text { oocytes with } \\
\text { spermatozoa bound }\end{array}$ & $\begin{array}{c}\text { Number of } \\
\text { spermatozoa per } \\
\text { oocyte }\end{array}$ & $\begin{array}{c}\text { Percentage of } \\
\text { oocytes with } \\
\text { spermatozoa bound }\end{array}$ \\
\hline FRE & 38 & $15.2(7.2-31.8)^{\mathrm{ac}}$ & $89^{\mathrm{a}}$ & $6.0(2.7-13.4)^{\mathrm{ad}}$ & $84^{\mathrm{a}}$ \\
\hline FRESS & 32 & $4.3(1.9-10.0)^{\mathrm{bc}}$ & $66^{\mathrm{b}}$ & $2.2(0.9-5.8)^{\mathrm{bc}}$ & $50^{\mathrm{b}}$ \\
\hline FRO & 108 & $5.7(2.7-11.7)^{\mathrm{bc}}$ & $81^{\mathrm{ab}}$ & $2.9(1.4-6.2)^{\mathrm{bd}}$ & $62^{\mathrm{b}}$ \\
\hline
\end{tabular}

Values presented are back-transformed estimated means with their upper and lower $95 \%$ confidence limits, and proportion of oocytes with spermatozoa bound to them $(\%)$.

FRE: oocytes from fresh ovaries; FRESS: salt-stored oocytes from fresh ovaries; FRO: oocytes from frozen ovaries.

${ }^{\mathrm{ab}}$ Values within a column that differ significantly $(P \leqslant 0.05)$ have different superscripts.

${ }^{\mathrm{cd}}$ Values within a row that differ significantly $(P \leqslant 0.05)$ have different superscripts.

dense zona pellucida, in which the fenestrations were less obvious, were also present. The thickness of the zona pellucida ranged from 3 to $6 \mathrm{~mm}$. The mean diameter of the oocytes was $79 \pm 12 \mu \mathrm{m}$.

Salt-stored oocytes from fresh ovaries (FRESS). Most oocytes showed larger fenestrations in the zona pellucida compared with the FRE group (Fig. 1c,d). The proportion of malformed oocytes was higher in this group than in the FRE group. The thickness of the zona pellucida was $0.3-1.0 \mathrm{~mm}$ and the oocytes had a mean diameter of $76 \pm 15 \mu \mathrm{m}$.

Unstored oocytes from frozen ovaries (FRO). The surface of the zona pellucida resembled that of oocytes in the FRESS group. Many oocytes had a zona pellucida with a sponge-like appearance and often the meshwork was wider than that of the fresh oocytes (Fig. 1e,f). The thickness of the zona pellucida was $6-8 \mu \mathrm{m}$. The mean diameter of the oocytes was $79 \pm 12 \mu \mathrm{m}$. Many oocytes in this group were malformed.

Salt-stored oocytes from frozen ovaries (FROSS). The oocytes in this group tended to be severely deformed and to have a concave shape (Fig. 1g). The ultrastructure of the zona pellucida in this group was similar that in the FRESS and FRO groups (Fig. 1h). There were broken oocytes in this group, although not in such a way that the thickness of the zona pellucida could be measured. The mean diameter of the oocytes was $75 \pm 13 \mu \mathrm{m}$.

\section{Discussion}

Salt storage of oocytes and the deep freezing of ovaries can both be used for the long-term preservation of oocytes. These techniques have been demonstrated in dogs as well as in other species. Salt-stored human and pig oocytes do not differ in their sperm-binding capacity compared with fresh oocytes (Yanagimachi et al., 1979; Liu et al., 1988; Kruger et al., 1991; Fazeli et al., 1995). The human zona pellucida retains biological and functional characteristics after 90 days in salt storage (Franken et al., 1991). However, in cows, the zonae pellucidae of salt-stored oocytes are less penetrable by spermatozoa than those of unstored oocytes (Chian et al., 1991). In dogs, Hay et al. (1997a) compared fresh COCs with salt-stored COCs and COCs from chilled ovaries, in terms of the number of spermatozoa attached to the zona pellucida. The sperm-binding rate did not differ between the fresh COCs and the COCs from chilled ovaries. However, there were significantly fewer spermatozoa bound to salt-stored COCs and it could not be established whether the decreased sperm-binding capacity was due to changes in the cumulus cells or changes in the zona pellucida. In the present study, the salt-storage of oocytes and deep freezing of ovaries both had a significantly negative effect on the sperm-binding potential of the zona pellucida, but the results indicate that the oocytes can be used in a sperm-zona pellucida binding assay.

With the gentle washing procedure, the proportion of oocytes that bound spermatozoa was significantly higher for the oocytes from fresh ovaries compared with the salt-stored oocytes from fresh ovaries. With the tough washing procedure, the proportion of oocytes that bound spermatozoa was greater for the oocytes from fresh ovaries compared with both the saltstored oocytes from fresh ovaries and oocytes from frozen ovaries. There was no significant difference in the proportion of oocytes that bound spermatozoa between the two groups of stored oocytes for either type of washing procedure. This indicates that oocyte storage has a substantially deleterious effect on the sperm binding capacity of many oocytes. However, the proportion of salt-stored oocytes from fresh ovaries that bound spermatozoa, that is $66 \%$ and $50 \%$ after gentle and tough washing, respectively, is higher than the proportion of salt-stored COCs binding spermatozoa $(36.5 \%)$ reported by Hay et al. (1997a). In humans, it has been reported that up to $25 \%$ of salt-stored zonae pellucidae will not bind any spermatozoa after relatively gentle washing (Liu et al., 1988). Species differences and differences in the test procedure, such as sperm concentration, are likely causes for this minor difference in the number of zona pellucida that do not bind spermatozoa. The number of spermatozoa bound to zonae pellucidae in the present study is similar to the results obtained in other studies using frozen-thawed canine semen and saltstored oocytes (Hay et al., 1997 a,b). Several factors affect the total number of spermatozoa bound to the zona pellucida, for 

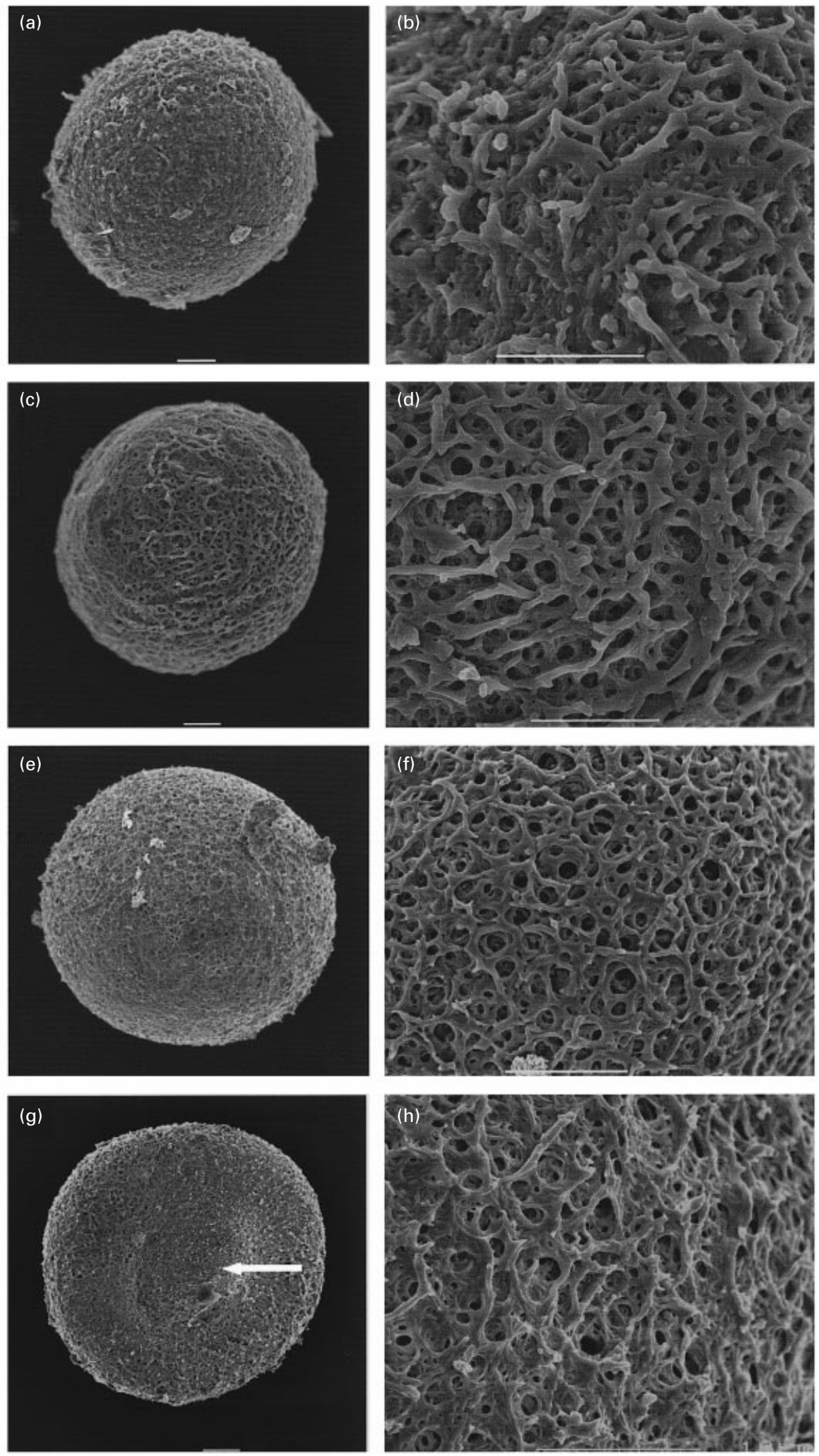

Fig. 1. Scanning electron micrographs of fresh and stored canine oocytes. Panoramic view $(\mathrm{a}, \mathrm{c}, \mathrm{e}, \mathrm{g})$; high magnification of the zona pellucida $(\mathrm{b}, \mathrm{d}, \mathrm{f}, \mathrm{h}) .(\mathrm{a}, \mathrm{b})$ Oocyte from a fresh ovary (FRE); (c,d) salt-stored oocyte from a fresh ovary (FRESS); (e,f) oocyte from a frozen ovary (FRO); (g,h) salt-stored oocyte from a frozen ovary (FROSS). The fenestrations of the zona pellucida are larger in the stored oocytes (c-h) than in the fresh oocyte $(\mathrm{a}, \mathrm{b})$. Note the malformation of the salt-stored oocyte from a frozen ovary (FROSS) visible on the panoramic view (g, arrow). Scale bars represent $10 \mu \mathrm{m}$. 
example sperm concentration and co-incubation time with the oocytes (Liu et al. 1988), whether fresh or frozen-thawed spermatozoa are used, whether the oocytes are fresh or stored, and the type of washing procedure used for the sperm-oocyte complexes. In additon, there may be species differences. There are thus several possible explanations for the fact that fewer spermatozoa bound to the zona pellucida in the present study compared with zona binding assays in humans (for example Franken et al., 1991, 1994; Kruger et al. 1991; Henkel et al. 1995).

The thickness of the zona pellucida in oocytes from fresh and frozen ovaries, 3-6 and 6-8 $\mu \mathrm{m}$, respectively, is less than that reported to be normal for mammals (10-20 $\mu \mathrm{m}$, Dunbar and Wolgemuth 1984; Keefe et al., 1997). The thickness of the salt-stored oocytes from fresh ovaries was considerably lower $(0.3-1.0 \mu \mathrm{m})$. Since the zona pellucida is a glycoprotein matrix (Dunbar and Wolgemuth, 1984) which is highly hydrated, dehydration could have been one cause for the thinness of the zonae pellucidae in the present study. The very thin zona pellucida of the salt-stored oocytes from fresh ovaries indicates that salt storage has a stronger dehydrating effect on the zona pellucida than deep freezing of ovaries. Another explanation for this finding could be that the zona pellucida of preantral follicles is thin.

The large variation within groups in the morphology of the oocytes was expected, considering the change in appearance of the zona pellucida in other species during maturation and degeneration. In humans, mice and goats, the surface of the zona pellucida changes during maturation of the oocyte (Familiari et al., 1988; Calafell et al., 1992; Villamediana et al., 1999). The mesh arrangement of the fibrous network was wider for mature oocytes, and it was suggested that a zona pellucida with an amorphous smooth surface corresponded to degenerating oocytes. In cows, no marked changes in the mesh structure were found during early in vitro maturation, whereas the fibrous network became finer and the meshes and holes shallower at the end of maturation (Suzuki et al., 1994). However, Magerkurth et al. (1999) could not establish any relationship between the structure of the zona pellucida and oocyte maturation or sperm binding pattern in humans. In mice, the structure of the zona pellucida changes with the age of the ovum (Longo 1981) as well as the age of the female (Nogués et al., 1988). In the present study, oocytes were recovered by slicing the ovary and thus were all immature. Therefore, the maturation stage of ovarian oocytes in bitches could only have explained a small amount of the variation in the morphology of the zona pellucida in the present study. The results did not provide any evidence that the age of the bitch affected the structure of the zona pellucida. However, the study was not designed to evaluate such an effect.

Marked decreases in the size and number of meshes in the zona pellucida after fertilization have been demonstrated in cows (Suzuki et al., 1994), whereas no changes in the zona pellucida were observed during fertilization in mice or hamsters (Phillips and Shalgi, 1980). In humans, Familiari et al. (1989) and Motta et al. (1991) reported no structural changes in the zona pellucida after in vitro fertilization. Familiari et al. (1992) reported no changes in the outer zona pellucida after fertilization, whereas the inner surface presented areas in which the filaments had fused. In contrast, other studies have reported that fertilization causes major ultrastructural changes in the zona pellucida in humans. Nikas et al. (1994) reported that, after fertilization, the porous zona pellucida with several superimposed layers of ring-shaped structures is transformed into a compact structure in which the ring-shaped structures appear to have melted and the pores are obscured by an amorphous material. These changes were thought to represent the zona reaction. Thus, changes in the morphology of the zona pellucida during maturation and after fertilization indicate that the ultrastructure of the zona pellucida might affect sperm binding capacity. This is further supported by the findings of Familiari et al. (1988), who reported that the degree of sperm binding to a spongy zona pellucida was higher than the degree of binding to a zona pellucida with a smooth surface, and that sites of sperm head attachment corresponded to the holes within the spongy framework. In the present study, the ultrastructure of the zona pellucida tended to differ between the oocytes from fresh ovaries and those in the other groups (FRESS, FRO and FROSS). Stored oocytes tended to have larger fenestrations and thinner filaments than fresh oocytes. The ultrastructure of the zona pellucida was similar for salt-stored oocytes from fresh and frozen ovaries and the unstored oocytes from frozen ovaries, although the salt-stored oocytes from frozen ovaries were more often malformed. Thus freezing and salt storage had similar effects on the ultrastructure of the zona pellucida. Although the larger meshwork of the zona pellucida of unstored oocytes has been ascribed to increased sperm binding, the ultrastructural changes in the stored zonae pellucidae in the present study are probably a reflection of damage caused during storage, leading to the reduced sperm binding potential of stored oocytes.

Lynham and Harrison (1998) suggested that, in pigs, the strength of sperm binding to the zona pellucida, measured as resistance to rinsing, depends on the physical orientation of the spermatozoa rather than on the degree of specificity of the binding. Lynham and Harrison (1998) based this hypothesis on the fact that they did not find any relationship between acrosomal status and binding strength. Consequently, they also questioned the relevance of a gentle rinsing procedure aimed at removing loosely bound spermatozoa. In the present study, the significant difference in sperm binding capacity between fresh and stored zonae pellucidae was observed after both the gentle and the tough washing procedures. Therefore, even though the relevance of the washing procedure might be questionable, it consistently discerned a difference in sperm binding potential between fresh and stored zonae pellucidae. The pronounced fenestrations of the zonae pellucidae of stored oocytes might affect the physical orientation of the spermatozoa, and thus the strength of the binding. In the salt-stored oocytes, the number of spermatozoa that remained bound after the tough washing procedure was not significantly lower than the number remaining after the gentle washing. This finding indicates that spermatozoa were able to bind to the salt-stored oocytes so strongly that they were not affected by the pipetting procedure.

In conclusion, sperm binding capacity was significantly greater in fresh compared with stored oocytes; the type of washing procedure did not affect this relationship. Of the two storage methods evaluated, deep freezing of ovaries appears to be a better method than salt storage of oocytes. Stored oocytes had a zona pellucida with a wider meshwork 
and larger fenestrations compared with fresh oocytes, but there was a large intra-group variation. The morphological changes associated with storage might explain, at least in part, the difference in sperm binding capacity. The potential for using stored canine oocytes for testing sperm function should be assessed in further studies.

The authors thank Ph. Lic. U. Engstrand for help with the statistical analyses, H. Ekwall for excellent preparation of the material for electron microscopy, and A. Jansson for technical assistance. L. Abersten, M. Ederoth and A. Gerentz Bohlin at the Department of Small Animal Clinical Sciences are thanked for help in collecting ovaries. Financial support was received from The Swedish Council for Forestry and Agricultural Research.

\section{References}

Altman D (1991) Practical Statistics for Medical Research Chapman and Hall, London

Andrews JC, Howard JG, Bavister BD and Wildt DE (1992) Sperm capacitation in the domestic cat (Felis catus) and leopard cat (Felis bengalensis) as studied with a salt stored zona pellucida penetration assay Molecular Reproduction and Development 31 200-207

Boatman DE, Andrews JC and Bavister BD (1988) A quantitative assay for capacitation: evaluation of multiple sperm penetration through the zona pellucida of salt-stored hamster eggs Gamete Research 19 19-29

Calafell JM, Nogués C, Ponsà M, Santaló J and Egozcue J (1992) Zona pellucida surface of immature and in vitro matured mouse oocytes: analysis by scanning electron microscopy Journal of Assisted Reproduction and Genetics 9365-372

Chian RC, Niwa K and Okuda K (1991) In vitro penetration of zona pellucida of salt-stored bovine oocytes before and after maturation by frozen-thawed spermatozoa Theriogenology 36 209-219

Dunbar BS and Wolgemuth DJ (1984) Structure and function of the mammalian zona pellucida, a unique extracellular matrix Modern Cell Biology 3 77-111

Familiari G, Nottola SA, Micara G, Aragona C and Motta PM (1988) Is the sperm-binding capability of the zona pellucida linked to its surface structure? A scanning electron microscopic study of human in vitro fertilization Journal of In Vitro Fertilization and Embryo Transfer 5 134-143

Familiari G, Nottola SA, Micara G, Aragona C and Motta PM (1989) Human in vitro fertilization: the fine three-dimensional architecture of the zona pellucida Progress in Clinical Biological Research 296 335-344

Familiari G, Nottola SA, Macchiarelli G, Micara G, Aragona C and Motta PM (1992) Human zona pellucida during in vitro fertilization: an ultrastructural study using saponin, ruthenium red, and osmiumthiocarbohydrazide Molecular Reproduction and Development 32 51-61

Fazeli AR, Holt C, Steenweg W, Bevers MM, Holt WV and Colenbrander B (1995) Development of a sperm hemizona binding assay for boar semen Theriogenology 44 17-27

Franken DR, Oosthuizen WT, Cooper S, Kruger TF, Burkman LJ, Coddington CC and Hodgen GD (1991) Electron microscopic evidence on the acrosomal status of bound sperm and their penetration into human hemizonae pellucida after storage in a buffered salt solution Andrologia 23 205-208

Franken DR, Kruger TF, Oehninger SC, Kaskar K and Hodgen GD (1994) Sperm binding capacity of human zona pellucida derived from oocytes obtained from different sources Andrologia 26 277-281

Hay MA, King WA, Gartley CJ, Leibo SP and Goodrowe KL (1997a) Canine spermatozoa - cryopreservation and evaluation of gamete interaction Theriogenology 48 1329-1342

Hay MA, King WA, Gartley CJ, Leibo SP and Goodrowe KL (1997b) Effects of cooling, freezing and glycerol on penetration of oocytes by spermatozoa in dogs Journal of Reproduction and Fertility Supplement 51 99-108

Henkel R, Cooper S, Kaskar K, Schill W-B, Habenicht U-F and Franken DR (1995) Influence of elevated $\mathrm{pH}$ levels on structural and functional characteristics of the human zona pellucida: functional morphological aspects Journal of Assisted Reproduction and Genetics 12 644-649

Ivanova M, Mollova M, Ivanova-Kicheva MG, Petrov M, Djarkova TS and Somlev B (1999) Effect of cryopreservation on zona-binding capacity of canine spermatozoa in vitro. Theriogenology 52 163-170

Keefe D, Tran P, Pellegrini C and Oldenbourg R (1997) Polarized light microscopy and digital image processing identify a multilaminar structure of the hamster zona pellucida Human Reproduction 12 1250-1252

Kruger TF, Oehninger S, Franken DR and Hodgen GD (1991) Hemizona assay: use of fresh versus salt-stored human oocytes to evaluate sperm binding potential to the zona pellucida Journal of In Vitro Fertilization and Embryo Transfer 8 154-156

Littell R, Milliken G, Stroup W and Wolfinger R (1996) SAS ${ }^{\circledR}$ System for Mixed Models SAS ${ }^{\circledR}$ Institute Inc., Cary, NC

Liu DY, Lopata A, Johnston WIH and Baker HWG (1988) A human sperm-zona pellucida test using oocytes that failed to fertilize in vitro. Fertility and Sterility $\mathbf{5 0} 782-788$

Longo FJ (1981) Changes in the zonae pellucidae and plasmalemmae of aging mouse eggs Biology of Reproduction 25 399-411

Lynham JA and Harrison RAP (1998) Use of stored pig eggs to assess boar sperm fertilizing functions in vitro. Biology of Reproduction 58 539-550

Magerkurth C, Töpfer-Petersen E, Schwartz P and Michelmann HW (1999) Scanning electron microscopy analysis of the human zona pellucida: influence of maturity and fertilization on morphology and sperm binding pattern Human Reproduction 14 1057-1066

Mahi CA and Yanagimachi R (1978) Capacitation, acrosome reaction, and egg penetration by canine spermatozoa in a simple defined medium Gamete Research 1 101-109

Mayenco-Aguirre AM and Pérez Cortés AB (1998) Preliminary results of hemizona assay (HZA) as a fertility test for canine spermatozoa Theriogenology 50 195-204

Motta PM, Familiari G, Nottola SA, Micara G and Aragona C (1991) Microstructural events of human egg investments during in vitro fertilization. Ultrastructure of the zona pellucida and cumulus oophorus Bulletin de l'Association des Anatomistes 75 89-91

Nikas G, Paraschos T, Psychoyos A and Handyside AH (1994) The zona reaction in human oocytes as seen with scanning electron microscopy Human Reproduction 11 2135-2138

Nogués C, Ponsà M, Vidal F, Boada M and Egozcue J (1988) Effects of aging on the zona pellucida surface of mouse oocytes Journal of In Vitro Fertilization and Embryo Transfer 5 225-229

Phillips DM and Shalgi R (1980) Surface architecture of the mouse and hamster zona pellucida and oocyte Journal of Ultrastructure Research 72 1-12

Rota A, Ström B, Linde-Forsberg C and Rodriguez-Martinez H (1997) Effects of Equex STM paste on viability of frozen-thawed dog spermatozoa during in vitro incubation at $38^{\circ} \mathrm{C}$ Theriogenology 47 1093-1101

Suzuki H, Yang X and Foote RH (1994) Surface alterations of the bovine oocyte and its investments during and after maturation in vitro. Molecular Reproduction and Fertility 38 421-430

Villamediana P, Ruttlán J, López-Béjar MA, Vidal F and Paramio MT (1999) Changes in zona pellucida surface after in vivo and in vitro maturation of caprine oocytes Reproduction in Domestic Animals 34 417-421

Yanagimachi R, Lopata A, Odom CB, Bronson RA, Mahi CA and Nicolson GL (1979) Retention of biologic characteristics of zona pellucida in highly concentrated salt solution: the use of salt-stored eggs for assessing the fertilizing capacity of spermatozoa Fertility and Sterility 31 562-574 